\title{
The role of visual attention in saccadic eye movements
}

\author{
JAMES E. HOFFMAN and BASKARAN SUBRAMANIAM \\ University of Delaware, Newark, Delaware
}

\begin{abstract}
The relationship between saccadic eye movements and covert orienting of visual spatial attention was investigated in two experiments. In the first experiment, subjects were required to make a saccade to a specified location while also detecting a visual target presented just prior to the eye movement. Detection accuracy was highest when the location of the target coincided with the location of the saccade, suggesting that subjects use spatial attention in the programming and/or execution of saccadic eye movements. In the second experiment, subjects were explicitly directed to attend to a particular location and to make a saccade to the same location or to a different one. Superior target detection occurred at the saccade location regardless of attention instructions. This finding shows that subjects cannot move their eyes to one location and attend to a different one. The results of these experiments suggest that visuospatial attention is an important mechanism in generating voluntary saccadic eye movements.
\end{abstract}

We selectively explore the visual panorama by means of fixations lasting about a quarter of a second interspersed with rapid changes of eye position lasting about $50 \mathrm{msec}$. The pattern of these fixations and the choice of where to send the eye next is not random but instead appears to be guided (Rayner \& Pollatsek, 1989). Yarbus (1967), for example, pointed out that the pattern of eye fixations that a given observer produces is influenced by properties of the scene as well as the goals and interests of the perceiver. Examples of this principle have been provided by many demonstrations that fixations in reading are influenced by properties of the text, such as word length (Rayner, 1975), as well as knowledge of the reader in the form of expectations, text schemas, and so on (Just \& Carpenter, 1987; Kowler, 1991).

What is the mechanism that chooses the destination of each subsequent saccade? A likely candidate is the spatial attention system, a mechanism that can operate within a fixation to selectively process information from different locations (Eriksen \& Hoffman, 1973, 1974; Hoffman, 1975; Hoffman \& Nelson, 1981; Posner, 1980; Posner, Nissen, \& Ogden, 1978). Allocating attention to a position in space results in faster and more accurate processing of luminance and form information in a region of space surrounding that location (Bashinski \& Bacharach, 1980; Downing, 1988; Hawkins et al., 1990; Hoffman \& Nelson,

This research was supported by University of Delaware Biomedical Research Grant and Army Research Office Contract DAAL03-86-k0080 to the first author and was submitted by the second author in partial fulfillment of the requirements for a Master of Arts degree. The authors are indebted to Lou Logan French for helpful comments on an earlier version of this paper. Portions of this paper were presented at the 32nd Annual Meeting of the Psychonomic Society, San Francisco, CA, November 1991. Reprint requests should be sent to J. E. Hoffman, Department of Psychology, University of Delaware, Newark, DE 19716.
1981). In addition to enhancing perceptual processing, attention may also be important in guiding "action systems," such as reaching (Allport, 1987, 1991). Because shifts of attention can occur much faster than changes in eye position (Hoffman, 1975), spatial attention can be used during one fixation to choose the location for the following fixation.

Indirect support for the claim that attention guides saccades is provided by a large literature showing that shapes are perceived faster and more accurately when they are near the target of an upcoming saccade. For example, McConkie and Rayner (1975) have used the moving-window paradigm to show that readers can perceive information about the length, shape, and identity of words in the periphery of the word they are currently fixating. This perceptual span is asymmetric (McConkie \& Rayner, 1976). For readers of English text, the span is larger on the right than on the left, consistent with the claim that eye movements to the right are preceded by allocation of attention in the same direction. In contrast, Hebrew text, which is read from right to left, leads to an asymmetry toward the left (Pollatsek, Bolozky, Well, \& Rayner, 1981). Similar results are found when subjects sequentially fixate a set of objects. Henderson, Pollatsek, and Rayner (1989) had subjects sequentially fixate four locations in which line drawings of objects could appear. In one condition, two objects were present on the screen at once, one in the currently fixated position and one in the position about to be fixated. This preview shortened the subsequent fixation on the object, relative to that when no preview was provided. In addition, this preview benefit was as large as that obtained in a condition in which all four objects were continuously available, suggesting that information is extracted primarily from the currently fixated object plus the one about to be fixated.

These results together suggest that attention and saccades are not independent. One version of the relationship 
between attention and saccades is the oculomotor readiness hypothesis (Klein, 1980; Rizzolatti, Riggio, Dascola, \& Umiltà, 1987), which holds that movements of both attention and saccades are mediated by the same neural circuitry. That is, when one attends or moves their eyes to a location, a set of commands is sent to the brain structures, such as the superior colliculus, that are responsible for oculomotor control. In the case of attention, however, the "go command" to actually execute the eye movement is inhibited, perhaps by circuitry involving areas of the frontal cortex (Guitton, Buchtel, \& Douglas, 1985).

The oculomotor readiness hypothesis makes two predictions: (1) preparing to make a saccade to a location should produce attentional enhancement at that location, and (2) attending to a location should result in fast saccades to that same location. Experiments designed to test these predictions have used some variant of a dual-task paradigm in which subjects are separately cued as to the location of the saccade and the likely location of a visual target that they must detect or identify. When the attention and the saccade are directed to different locations, the subject should be faced with a classic dual-task interference situation. If the saccade task is emphasized, the detection/identification task should suffer when the target and saccade location are different and the cue validly indicates the target location. On the other hand, when the detection/identification task is emphasized, saccades should be slow on the trials when attention is allocated to one location and the eyes must be sent to a different location. The experimental results testing these predictions have been mixed, perhaps due to various methodological problems (detailed below).

Klein (1980, Experiment 1) attempted to provide a direct test of the prediction that preparing a saccade to a location should enhance the detection of signals at that same location. Subjects were instructed to make a saccade in a particular direction whenever they detected the occurrence of an asterisk that could appear to the left or right of fixation. On some trials, a brief brightening of a dot on the left or right was presented instead of the asterisk. Subjects were to make a manual detection response to the dot. Suppose that the subject is prepared to move left and the target is presented to the left. If the subject is attending to the left location as a necessary component of programming a saccade to that location, then there should be an advantage for detecting probes in that location (Posner et al., 1978). In fact, Klein found no reduction in reaction times to probes presented in the location that was the target of a saccade, and he concluded that shifts of attention are not a necessary component of saccade preparation.

There are several features of Klein's data that suggest caution in accepting his conclusions. First, there is no independent evidence that subjects were, in fact, preparing a saccade to the designated location at the moment that the probe was presented. Saccade latency in dual-task blocks was about $100 \mathrm{msec}$ slower than that in the single-task control condition. This difference could be due to subjects' waiting for the stimulus to appear to determine the nature of their task on that trial. This strategy would obvi- ously preclude a test of the relationship between attention and saccades.

A larger dual-task increment can be seen in the reaction times for detecting the probe, and this increment raises a different interpretational difficulty. Pashler (1989) has shown that there are often delays in executing the second of two speeded responses to signals presented in close temporal proximity. In addition, factors (such as attention) that might normally speed the perceptual processing of the first stimulus may be ineffective under dual-task conditions because a delay in the response selection stage of the second task essentially masks the effects of variables affecting earlier processing stages. In Klein's experiments, only one response was required on a given trial, but subjects may have still adopted the strategy of primarily preparing to respond to the most likely task on a given trial (e.g., the saccade task in Experiment 1), producing large delays in the processing of the unexpected task (the detection task). Thus, the lack of an attentional effect of saccades in Klein's experiments may be due, at least in part, to "underadditivity" (Pashler, 1989), which can occur in dual-task reaction time studies. Similar comments apply to a recent replication and extension of Klein's original experiment reported by Klein and Pontefract (1994).

Remington (1980) reported a positive relationship between saccades and attention using a detection accuracy paradigm. Subjects were instructed to make a saccade to a peripheral transient and to detect a brief target flash presented on the same or opposite side as the transient. Detection accuracy was highest when the detection and saccade tasks were compatible. This result is difficult to interpret, however, because the cue used to initiate the saccade would be expected to capture attention automatically (Remington, Johnston, \& Yantis, 1992). Therefore, this result may tell us more about the attention-capturing properties of peripheral transients than the relation between attention and saccades. This is consistent with an additional experiment reported by Remington in which this relationship was eliminated when the saccade was cued by a central cue rather than a peripheral one. A further difficulty is that Remington used a percent hit measure, which is vulnerable to changes in criterion. In addition, he did not eliminate trials in which the eye movement was initiated prior to target onset, raising the possibility that, at least in some conditions, any attention effects that were observed may have been due to changes in the position of the target with respect to the fovea.

Many of these methodological difficulties were eliminated in a recent experiment reported by Shepherd, Findlay, and Hockey (1986). They presented a central cue that instructed subjects on both the direction of the saccade and the likely location of the target flash. They found that subjects were faster at detecting flashes on the same side as the saccade even when targets were more likely to have occurred on the opposite side of the display. Unfortunately, they left the probe on the screen until the subject responded. Because the mean saccade latencies were less than the mean manual reaction times, targets presented on 
the same side as the saccade could, at least on some trials, be foveated before the response. Thus, we do not know whether the advantage of probes presented at the target of the saccade is due to attentional effects or the difference between foveal and eccentric acuity differences.

The present set of experiments was designed to investigate the relation between attention and saccades with a dual-task methodology that eliminates the problems identified above. The direction of saccades either was kept fixed for an entire block of trials (Experiment 2) or was signaled well before the onset of the array that was used to detect shifts of attention (Experiment 1). Thus, the cue to shift the eyes need not be signaled by a visual signal, which itself can capture attention and affect the detection of the array target. The detection/identification task was a forced choice identification procedure without time pressure, eliminating the dual-task underadditivity that can occur with reaction time measures. Attention effects are measured with a brief visual display that is removed prior to the onset of the saccade.

Experiment 1 tested whether or not requiring subjects to make saccades to a location improved the identification of information in that location. The logic of the experiment is similar to the Henderson et al. (1989) study described earlier, except that we were measuring attention effects using accuracy in a shape discrimination task rather than fixation durations. No explicit attentional instructions were provided. Experiment 2 tested whether attention and saccades could be directed to different locations without mutual interference. Subjects made a saccade to one location and were instructed to attend to the same or a different location for the target identification task.

\section{EXPERIMENT 1}

Experiment 1 was designed to test whether or not making a saccade to a location is preceded by a shift of attention to that location. No attentional instructions were provided, so that Experiment 1 tested whether subjects choose to attend to a location that is the target of a saccade. The question of whether this attentional shift is obligatory was examined in the second experiment.

\section{Method}

Subjects. Five male and 2 female undergraduate students at the University of Delaware participated in the experiment as paid volunteers. All of them had normal or corrected-to-normal vision.

Apparatus and Stimuli. All visual information was presented on a Vectrix VXM color graphics monitor under control of an IBM AT computer except for the letters that composed the detection task. They were presented using a carousel slide projector equipped with a Uniblitz Model 310 high-speed shutter. The subjects placed their heads in a chinrest and viewed the computer screen through a beam splitter. Slides were projected onto a screen that, when viewed through the beam splitter, appeared to be superimposed on the computer screen. Eye movements were monitored with a Micromeasurements System 1200 camera-based eye-tracking system with a spatial resolution of $0.5^{\circ}$ of visual angle and a temporal resolution of $16.7 \mathrm{msec}$.
The fixation display consisted of a central plus sign $\left(0.36^{\circ}\right.$ visual angle) and four target locations/saccade destinations identified by open rectangles sized $0.8^{\circ} \times 0.6^{\circ}$. Rectangles appeared left, right, above, and below the fixation point. The distance between the fixation point and each of the target locations was $5^{\circ}$. Letters were chosen from the set E, F, L, and T and were $0.7^{\circ} \times 0.5^{\circ}$. The arrow used to indicate eye-movement direction was $0.5^{\circ}$ in length. The spot used as the target in eye-movement calibration was $0.36^{\circ}$. All stimuli were white and appeared against a black screen. The luminance of computer-drawn stimuli was $72 \mathrm{ml}$. The projected letters had a luminance of $180 \mathrm{ml}$. The subjects initiated each trial and made their detection responses using the standard PC mouse.

Procedure. Each session consisted of five blocks of trials. The first block was used to calibrate the eye-tracking system. The subjects were instructed to move their eyes from fixation to the four target locations as well as to locations halfway between the fixation point and the target locations. These measurements were made twice and were used to ensure that the subjects were making eye movements to the instructed locations. Saccade latency was defined as the first sample from the eye tracker with a deviation of a half degree from fixation. Saccade direction in subsequent blocks was determined by comparing the fixation location following the saccade with the fixation locations collected during the calibration block. The experimenter monitored the position of the pupil on the screen throughout the session and periodically adjusted the camera to ensure that the subject's pupil was "centered" and in good focus.

In the second block, the PEST procedure (Taylor \& Creelman, 1967) was used to estimate the stimulus duration used for the letter array for each subject in each session so as to produce about $80 \%$ correct target detection. The average duration across subjects was $11 \mathrm{msec}$. This block had a variable number of trials that ranged between 20 and 40 . In the remaining three blocks, the subjects performed each of the single-task control conditions (saccade and detection) and the dual-task combination. The order of these three blocks was counterbalanced across sessions for each subject.

In the dual-task block, the subjects fixated the central plus and initiated a trial by pressing a mouse button. An arrow was presented for $1,000 \mathrm{msec}$ pointing to one of the four rectangles that was to serve as the target of a saccade. The arrow display was terminated and followed by an interval chosen randomly from $500-2,000 \mathrm{msec}$. A tone was then presented $(500 \mathrm{~Hz}$ for $20 \mathrm{msec}$ ) that served as the signal to initiate the saccade. Following the tone at an SOA of 0,50 , or $100 \mathrm{msec}$, a four-letter array was presented, each letter occupying one of the four rectangles. This array contained three distracter letters (Es and Fs) and one target letter (a $T$ or an L). The subjects had to indicate which target had occurred after completing their eye movement. Feedback on detection accuracy was provided after each trial. The subjects were informed that a target letter was equally likely to appear at any one of the four target locations and, hence, it was not useful to attend to any one location selectively.

In dual-task blocks, there were four possible locations as destinations for the saccades, two possible target letters, four possible target locations, and three possible SOAs (interval between the tone and the stimulus onset), resulting in 96 trials in this block. On one fourth of the trials, the target occurred at the same position as the destination of the saccade. These were designated saccade-target match trials. All other trials were saccade-target mismatch trials.

The sequence of events in the eye-movement-only condition was similar except a target letter was not present in the array and a choice response was not required. The subjects were instructed to fixate on the central fixation point and, at the sound of a tone, execute a saccade to the cued location as quickly and accurately as possible. They were asked to press any button on the mouse after executing the saccade to end the trial. There were four possible locations as destinations for the saccades and three possible delays, producing 12 possible combinations. This set of 12 trials was repeated twice for a total of 24 trials. 
In the detection-only block, instead of the eye-movement cue (the central arrow), a no-eye-movement cue (a small circle around the fixation point) was presented at the start of each trial. This was designed to mimic any warning signal effects associated with the cue in the dual-task block. In the detection-only block, there were two possible target letters, four possible target locations, and three possible delays, yielding 24 trials in this block.

Each subject was run in six sessions within a period of 10 days. The first two sessions were considered practice, and data from these sessions were not analyzed.

\section{Results}

In the detection-only block, trials with detectable eye movements were rejected ( $1.5 \%$ of trials). In the movement-only and the dual-task blocks, a trial was rejected if (1) the eye-movement latency was less than $100 \mathrm{msec}$ $(0.5 \%$ of trials), (2) the eye-movement latency was more than two standard deviations above the mean latency for the subject ( $4 \%$ of trials), or (3) there was no eye movement or the eye movement was made to an incorrect location ( $21 \%$ of trials).

The principal question is whether making a saccade to a location improves target discriminabiiity for that location. Figure 1 shows target detection accuracy as a function of delay for the single- and dual-task conditions. The dual-task data have been separated according to whether the target occurred in the location that was the destination of a saccade (saccade-target match) or in a different location (saccade-target mismatch). Targets were detected best when they occurred in a location to which the subjects were about to move their eyes. Intermediate performance occurred in the detection-only condition when the subjects had no bias toward a particular location. Worst performance occurred in the dual-task condition when targets occurred in display positions that were not the destination of the saccade. Increasing the interval between the saccade and the presentation of the array produced a small decrease in target detection accuracy in dual-task conditions and an improvement in the detection-only condition. These effects of delay were small and nonsignificant.

These impressions were confirmed in a repeated measure analysis of variance (ANOVA), which showed a

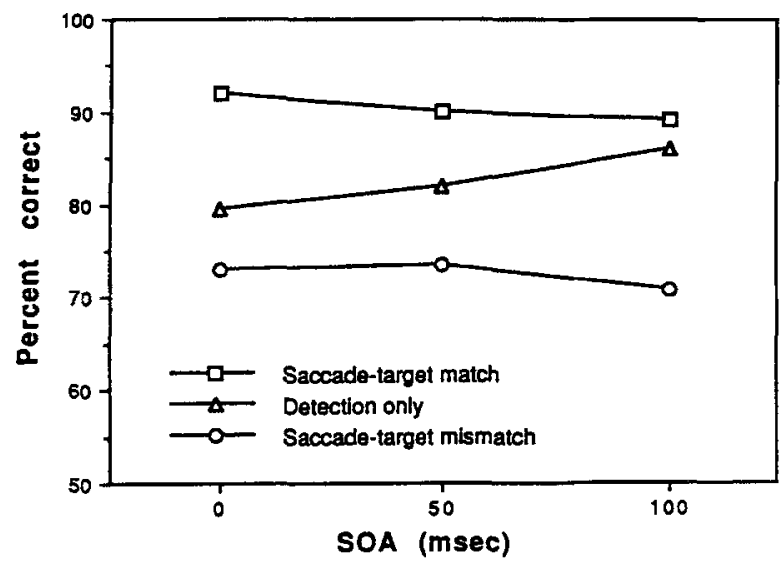

Figure 1. Mean target-detection accuracy in Experiment 1.

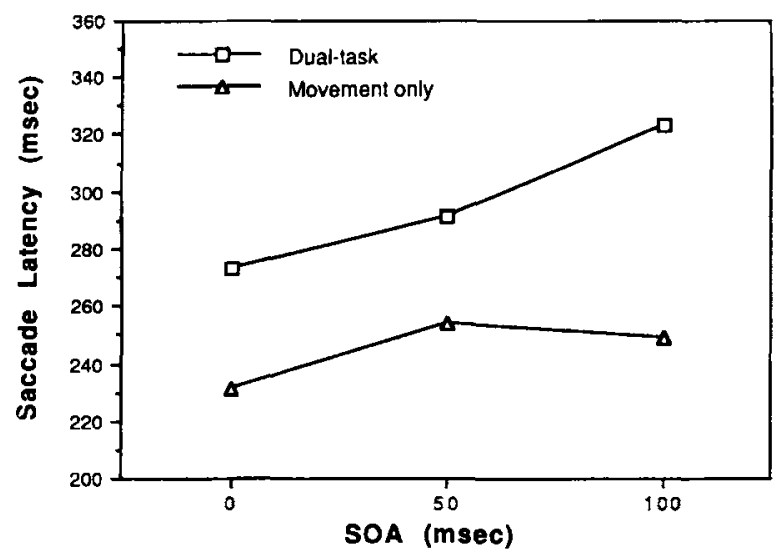

Figure 2. Mean saccadic latency in Experiment 1.

highly significant main effect of saccade-target match/mismatch, $[F(2,12)=147.63, p<.001]$. There was no significant main effect of delay $[F(2,12)=0.027, p=.97]$ or its interaction with saccade-target match/mismatch $[F(4,24)$ $=2.201, p>.09]$. A planned comparison of the means showed that the performance in the detection-only block was significantly inferior to the performance in the saccade-target match condition of the dual-task block $[F(1,6)=67.78, p<.001]$ but was significantly superior to the performance in the saccade-target mismatch condition of the dual-task block $[F(1,6)=94.48, p<.001]$.

The eye-movement latencies from the movement-only and dual-task blocks are shown in Figure 2. There was a significant slowing of saccades in dual-task conditions relative to the single-task latency $[F(1,6)=6.27, p<.05]$, consistent with the claim that the letter detection and saccade tasks share some limited attentional system. In addition, there was a progressive slowing in the saccade latency as the letter array was delayed $[F(2,12)=11.79, p<$ $.01]$, as well as an interaction of SOA and task $[F(2,12)=$ $4.27, p<.05]$. The form of the interaction suggests that the subjects may have delayed executing the saccade until the arrival of the array, at least on some of the trials.

\section{Discussion}

The goal of Experiment 1 was to evaluate the claim that attention and saccades reflect independent processes. Contrary to this hypothesis, we found that making a saccade to a location improves the detectability of information presented in that location. This is the expected result if a shift of visual attention precedes saccadic eye movements. It should be noted that this improvement in detection was not due to the subjects' foveating the target letter on these trials, because the stimulus array was shown and removed well before the start of the saccade on each trial. The subjects were aware of the fact that the target letter could appear in each of the target locations with equal probability and that they should simultaneously attend to all target locations to maximize their detection performance. In spite of this, the results show that the subjects attended to the location that was the target of a saccade. 
Apparently, programming or execution (Klein, Kingstone, \& Pontefract, 1992) of voluntary saccades is accompanied by the same attentional enhancement that is found when subjects are explicitly instructed to pay attention to a location in space.

This result is consistent with the general finding in other studies that used cost-benefit paradigms to study the allocation of visual attention to different spatial locations (Downing, 1988; Hawkins et al., 1990; Posner et al., 1978). In these studies, subjects' performance in a target detection task benefited from advance knowledge of the target location and suffered if the target appeared at an unexpected location. The benefits were observed either as a decrease in reaction time or as an increase in target detection accuracy, and costs were observed either as an increase in reaction time or as a decrease in target detection accuracy. A similar pattern of costs and benefits was observed in this experiment when the subjects were about to make an eye movement to a location, even though they knew that targets were no more likely to occur in that location than in any other.

We also studied the effects of the time interval between the signal to initiate the saccade and the onset of the letter array in an attempt to examine the time course of attentional allocation preceding a saccade. We found no significant difference in detection performance between the three delay conditions $(0,50$, and $100 \mathrm{msec})$. In fact, the data show a significant effect of saccade-target match/ mismatch even in the 0-msec delay condition, suggesting that the orienting of attention to the saccade destination location may have occurred prior to the presentation of the saccade initiation signal. Thus, the destination of the saccade may be programmed with the aid of the spatial attention system, with the final "go" signal being provided by the onset of the tone.

A straightforward interpretation of the effects of delaying the onset of the letter array depends on subjects' initiating the saccade at the onset of the tone (since delay is measured relative to the tone onset). This requirement was not strictly met. The saccadic latencies showed an increase with increasing delay. There was approximately a $25-\mathrm{msec}$ increase in saccadic latency for every 50 -msec increase in the delay between tone and array onset (see Figure 2). This suggests that the subjects may have used some combination of the tone and the visual array as the effective signal for initiating the saccade. This may be one reason that the delay variable had so little effect on detection performance. To the extent that the subjects waited for the appearance of the array to initiate their saccade, our delay manipulation was ineffective in mapping the temporal course of attentional allocation.

In conclusion, the results of Experiment 1 suggest a link between attention and saccadic eye movements. When subjects move their eyes to a location in space, they attend to that location prior to the saccade. Experiment 2 investigated whether this link is optional or obligatory.

\section{EXPERIMENT 2}

In Experiment 2, subjects were given explicit instructions to attend to a particular location, which might or might not have been the same as the destination of their saccade (see Figure 3). The question is whether subjects can use the attentional cue to attend to one location while making a saccade to a different one. The design and logic were similar to those used by Shepherd et al. (1986). The major difference is that we presented instructions relevant to the two tasks separately and well before the required eye movement. This situation was expected to provide optimal conditions for a separation of attention and saccades if such a separation is possible. In addition, the letter array used to measure allocation of attention was briefly presented and always removed before the onset of the saccade.

\section{Method}

Subjects. Four male and 3 female undergraduate students at the University of Delaware participated in the experiment as paid vol-

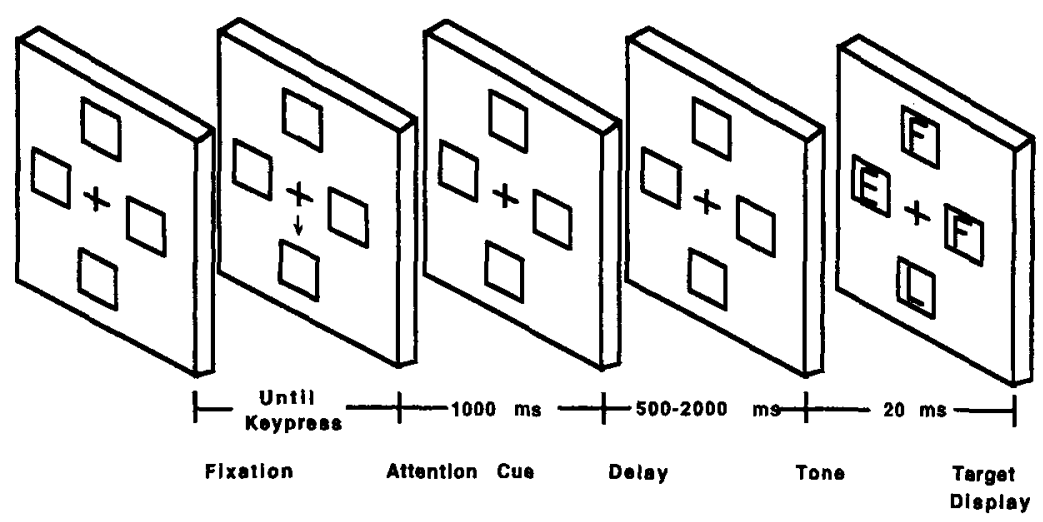

Figure 3. The sequence of events in dual-task trials of Experiment 2. In this example, the subjects were instructed to make saccades to the right box. The attention cue indicates that the bottom box is the likely location of the target ( $\operatorname{Lan}$ or $a \mathrm{~T}$ ). The target does occur in the cued location, making this a cue-target match trial. The cue and saccade instructions do not match (cue-saccade mismatch). 
unteers. Five of these subjects had also served in Experiment 1. All of them had normal or corrected-to-normal vision.

Apparatus and Stimuli. The apparatus and stimuli were identical to those used in Experiment 1.

Procedure. Each session consisted of five blocks of trials. The first block was used to calibrate the eye-tracking equipment, and the second block was used to estimate the stimulus duration required for approximately $80 \%$ performance. The procedure was identical to that used in Experiment 1. The display duration, averaged over sessions and subjects, was $12 \mathrm{msec}$. The last three blocks consisted of the detection-only, movement-only, and dual-task blocks (presented in that order). These blocks followed a procedure similar to that employed in Experiment 1, with the following major exception. In Experiment 1 , the arrow presented before the array indicated the direction for eye movements; in Experiment 2, the direction for eye movements was specified at the beginning of the session and remained the same for the entire session.

The arrow was used in this experiment to indicate the likely location of the target and had a validity of $75 \%$ (i.e., the target occurred in the cued location on $75 \%$ of the trials). On the remaining $25 \%$ of the trials, the target occurred in each of the uncued display positions equally often. Therefore, the location of the saccade and the location of the target in the array matched on $25 \%$ of the trials, and there was no correlation between target location and saccade direction.

In the detection-only block, the subjects fixated on the central fixation cross and initiated each trial by pressing a mouse button. A central cue (an arrow extending from the fixation cross) was presented for 1,000 msec. The subjects were informed that the cue had a $75 \%$ probability of correctly predicting the target location and were advised to divide their attention accordingly to maximize detection performance. The offset of the cue was followed by a randomly chosen time interval between 500 and $2,000 \mathrm{msec}$. A 20 -msec tone was then presented, and the stimulus array was displayed immediately after the offset of the tone. This tone was irrelevant to the task in this block. Since it was used as a saccade initiation signal in the fourth and fifth blocks, the tone was retained in this block to maintain the equivalence of events in all three blocks. As in Experiment 1 , the trials in this block ended when the subjects gave their detection response by pressing the appropriate mouse button indicating whether the target was an $\mathrm{L}$ or a $\mathrm{T}$. The eye position was recorded for each trial from the start of the trial to 300 msec after the end of the trial. The subjects were instructed to maintain fixation until their response.

Feedback on detection accuracy was provided after each trial. There were 24 trials in this block. Each of the four display positions was cued equally often. The target occurred in the cued position on $75 \%$ of the trials. On the uncued trials, the target occurred in each of the three uncued positions equally often.

Procedure in the dual-task block was similar, except that the subjects were instructed to make a saccade to one of the four fixation boxes when they heard the tone. The saccade direction remained fixed throughout the session and was counterbalanced across sessions and subjects. As in Experiment 1, the instructions to the subjects emphasized the speed and accuracy of saccades and accuracy of target detection over the speed of detection response. At the end of the trial, the accuracy of response, saccadic latency for that trial, and the mean saccadic latency in the movement-only block were displayed to the subject as feedback. The subjects were told to adjust their saccade latency so that their trial-by-trial saccadic latency was close to the mean saccadic latency from the movement-only block. They gave their detection response after completing the saccade.

Each of the four display positions was cued equally often, with each target appearing in the cued position on $75 \%$ of the trials. Targets also appeared in each display position equally often on invalid cue trials, yielding a total of 96 trials in this block. There was no correlation between the direction of saccade and the position of the target in the array.

In the movement-only block, the subjects made saccadic eye movements from the central fixation point to a specified location. The time course of events in this block was similar to that of the last block. However, instead of the arrow cue, a small circle around the fixation point was used, and there was no target letter present in the stimulus array. The subjects were told to ignore the stimulus array. The subjects were instructed to fixate on the central fixation point and, when they heard the tone, they were to execute a saccade to the specified location as quickly and accurately as possible. They were asked to press any button on the mouse after executing the saccade to end the trial. Eye movement was recorded for each trial from the start of the trial to $300 \mathrm{msec}$ following the target detection response. There were 24 trials in this block. The latency of saccadic eye movements from this block was used as a baseline measure to compare with the dual-task data.

Each subject was run in six sessions at the rate of one session per day. The first two sessions were intended as practice and, during these sessions, their performance was closely monitored and additional feedback was given to familiarize them with the task. The data from the last four sessions were used in the analysis.

\section{Results}

A trial was excluded from the analysis if (1) the eyemovement latency was less than $100 \mathrm{msec}$ ( $3 \%$ of trials), (2) the eye movement was more than two standard deviations above the mean latency for the subject ( $5 \%$ of trials), or (3) there was no eye movement or the eye movement was made to an incorrect location ( $15 \%$ of trials).

The mean target detection accuracy data from the dualtask and detection-only blocks are shown in Table 1. Consider first the detection-only data. There was a large attentional cuing effect, with detection accuracy being about $12 \%$ better when the target appeared in the cued location than when it appeared in the uncued locations. This result replicates the findings of a large number of studies showing that subjects can selectively attend to one location in a multielement array with a resulting increase in forced choice detection accuracy (e.g., Cheal \& Lyon, 1989; Lyon, 1990). More importantly, it shows that our manipulation of attention in this study was effective. A

Table 1

Mean Percent Target Detection Accuracy in Experiment 2

\begin{tabular}{lccc}
\hline & \multicolumn{2}{c}{ Dual-Task Block } \\
\cline { 2 - 3 } Condition & Detection-Only Block & $\begin{array}{c}\text { Saccade-Target } \\
\text { Match }\end{array}$ & $\begin{array}{c}\text { Saccade-Target } \\
\text { Mismatch }\end{array}$ \\
\hline $\begin{array}{c}\text { Cue-target } \\
\text { match }\end{array}$ & 79.3 & 86.6 & 73.4 \\
$\begin{array}{c}\text { Cue-target } \\
\text { mismatch }\end{array}$ & 66.93 & 86.4 & 70.4 \\
\hline
\end{tabular}


very different pattern of results is apparent in the dual-task condition. When the target occurred in the location that was the destination of the saccade (saccade-target match), target detection was uniformly high regardless of whether the cue was directing attention to that location or to a different one. When the saccade was directed to a location that did not contain a target (saccade-target mismatch), detection performance was uniformly low and again independent of whether the cue was validly indicating the position of the target.

Separate repeated measures ANOVAs were performed on detection-only and dual-task conditions. In the dualtask condition, there was a significant difference between the saccade-target match and saccade-target mismatch conditions $[F(1,6)=119.36, p<.001]$. However, there was no significant difference between the cue-target match and cue-target mismatch conditions $[F(1,6)=1.18, p>$ .31]. In contrast, the target detection accuracy in the detection-only block was significantly better in the cuetarget match condition than in the cue-target mismatch condition $[F(1,6)=108.65, p<.001]$.

The average eye-movement latencies from the movement-only block and the cue-saccade match and cuesaccade mismatch conditions of the dual-task block are shown in Table 2. An ANOVA of the data showed a significant difference between these three conditions $[F(2,12)=$ $6.43, p<.02]$. A planned comparison of the means showed a significant difference between the cue-saccade match and cue-saccade mismatch conditions $[t(6)=3.03, p \leq$ $.025]$. In addition, latency in the cue-saccade match condition was faster than latency in the movement-only condition $[t(6)=3.0, p<.025]$. The cue-saccade match and movement-only conditions did not differ $[t(6)=0.68, p>$ $.5]$. These analyses show that the subjects executed their saccades faster when the direction of the saccade agreed with the attention cue than when there was a conflict.

\section{Discussion}

Experiment 2 attempted to establish optimal conditions for allowing subjects to separate attentional allocation from saccadic eye movements. The direction and location of the saccade were the same for an entire session. The cue to orient attention was provided several seconds prior to the display. Nonetheless, our data strongly support the claim (Shepherd et al., 1986) that subjects first attend to a location before they move their eyes to it. Targets were detected better when they occurred at the destination of a saccade independent of where the subjects were instructed to attend. In other words, the direction in which the subject was making an eye movement determined the allocation of attention, not our attentional cuing instructions, which, otherwise, were quite effective in the detection-only condition.

Table 2

Mean Saccadic Latency in Experiment 2

\begin{tabular}{ccc}
\hline $\begin{array}{l}\text { Cue-Saccade } \\
\text { Match }\end{array}$ & Movement Only & $\begin{array}{c}\text { Cue-Saccade } \\
\text { Mismatch }\end{array}$ \\
\hline 232 & 243 & 246 \\
\hline
\end{tabular}

Note-All values are given in milliseconds.
The lack of any effect of the cue might suggest that the subjects were simply ignoring the detection task and concentrating on making eye movements. However, several aspects of our data argue against this. First, detection accuracy was quite high in dual-task conditions when the subjects were prepared to make a saccade to the same location occupied by a target letter. In fact, detection accuracy was higher here than in the detection-only condition with valid cues. Apparently, making a saccade to a location produces a greater allocation of spatial attention to the saccade target location than does a $75 \%$ valid attention cue.

A second relevant finding is that saccades were slightly (and significantly) faster when they were made in the same direction as the attention cue, relative to when they conflicted. ${ }^{1}$ This finding shows that the subjects were not ignoring the attention cue but were in a dual-task interference situation. Recall that our instructions to the subjects emphasized that eye movements should be made as fast as those observed in the single-task eye-movement condition. Apparently, the subjects chose an attention allocation strategy emphasizing the saccade task, resulting in target detection being dominated by the location of the intended saccade and not the attention cue. Kowler, Anderson, Dosher, and Blaser (in press) found that there are a variety of points along this tradeoff function that subjects can adopt. Increasing emphasis on the detection task would result in larger cuing effects and greater increases in saccade latency.

\section{GENERAL DISCUSSION}

The present study has shown that a saccade executed to a peripheral location in the visual field involves orienting of attention to that location, prior to the actual execution of the saccade. The inability to orient attention to one location and simultaneously execute a saccade to another location suggests that orienting of attention may be an essential component of preparing and/or executing saccades. Experiment 1 showed a link between attention and saccades when subjects are free to orient their attention as they wish. Experiment 2 showed that the link between attention and saccades is obligatory. That is, subjects have difficulty moving their eyes to one location and attending to another, even when instructed to do so. In addition, this result holds even when subjects know the destinations of attention and saccades well ahead of time, providing ample time to resolve any conflicts in programming. Similar conclusions seem to hold for the relationship between attention and pursuit eye movements (Kowler, 1985).

There are, of course, a variety of ways to conceptualize the relationship between movements of attention and the eyes. Perhaps the simplest is to assume that saccades and pursuit eye movements require the involvement of the attentional system to choose which stimuli in the field are to gain control of the oculomotor system. In this view, the eye-movement system is but one of many perceptual and response systems that benefit from attentional selection. A more radical view, also consistent with our results, is the 
oculomotor readiness hypothesis, which holds that movements of attention depend on the activation of brain structures that are intimately involved in moving the eyes. This position has been supported in recent years by Rizzolatti (e.g., Rizzolatti et al., 1987; Rizzolatti, Riggio, \& Sheliga, 1994), who calls it the premotor theory of attention.

The premotor theory of attention makes several predictions. First, damage to oculomotor centers in the brain ought to produce corresponding deficits in covert orienting, and, indeed, there seems to be good support for this proposition. Rafal, Posner, Friedman, Inhoff, and Bernstein (1988) studied patients with progressive supranuclear palsy, a degenerative disorder that attacks the superior colliculus (SC), among other structures. Consistent with the role of SC in producing saccades, these patients display a deficit in making voluntary saccades, particularly in the vertical direction. Rafal et al. used a peripheral cuing paradigm and observed similar difficulties in patients' covert orienting of attention in the vertical direction. Similarly, Desimone, Wessinger, Thomas, and Schneider (1989) reported that small focal lesions in $\mathrm{SC}$ in monkeys produced deficits in covert orienting to stimuli occurring in the receptive fields of the lesioned cells.

A second prediction of the premotor theory of attention is that spatially selective activity should be observed in oculomotor brain areas during selective attention. Wurtz, Goldberg, and Robinson (1980, 1982) originally observed enhanced activity in SC cells preceding a saccade to a stimulus located in the receptive field of the cell. This enhancement effect occurred only when the monkey actually made an eye movement, and it was absent when attention was allocated for another purpose, such as making a reaching movement.

More recent studies, however, have shown enhancement of cells in SC even when the animal does not make an eye movement. Gattas and Desimone (1992) presented relevant and irrelevant stimuli to monkeys in a matchingto-sample task. Cell activity of SC cells was enhanced when the relevant stimulus was in the receptive field as opposed to the irrelevant one. Presumably, the animal's attention to the relevant stimulus produced or was mediated by enhancement of firing in SC cells. The latter possibility was supported by the finding that direct stimulation of cells in the superficial layer of the SC with receptive fields at the location of the distracter reduced behavioral performance, suggesting that $\mathrm{SC}$ activity at a particular location tends to program attention to be allocated to that location. These findings offer direct support for the proposition that at least some structures known to be involved in the control of eye movements appear to also play a role in directing attention.

Behavioral investigations of the premotor theory have produced a somewhat mixed set of findings. Rizzolatti et al. (1987) suggested that the meridian effect offers support for the premotor theory of attention. Their subjects made speeded detection responses to light flashes whose likely positions were indicated by a central precue. On invalid trials, they found that costs were greater when the cued and target positions were on opposite sides of the vertical or horizontal meridians than when they were on the same side. If movements of attention from the cued position to the target position are carried out by programming the oculomotor system, then asymmetries in moving the eyes in certain directions would also be reflected in movements of attention even though no saccades are involved. Rizzolatti et al. suggested that when cued and target positions fall on opposite sides of a meridian, saccade programming involves a change in direction rather than just a change of amplitude, accounting for the greater costs when crossing a meridian.

Reuter-Lorenz and Fendrich (1992) provided a direct test of these claims by investigating the meridian effect for both saccadic and manual detection reaction times. With central precues, they found that both saccades and detection responses showed the meridian effect, as predicted by the premotor theory. With peripheral precues, however, there were no meridian effects for either task. At the very least, these results suggest that central and peripheral cues may rely on different mechanisms for directing attention and that oculomotor mechanisms may play a greater role in endogenous movements of attention than in exogenous movements.

The present results, as well as similar findings reported by Kowler et al. (in press) and Schneider and Deubel (1995), show that there is a close relationship between attention and saccades. Together with Kowler's (1985) work showing a similar relationship between pursuit eye movements and attention, they suggest that oculomotor mechanisms rely on attention for some aspect of the control of eye movements. Klein et al. (1992) suggest that attention is important in the execution of eye movements but plays no role in the planning of eye movements. Further experiments are required to sort out these possibilities. The ancillary position taken by oculomotor readiness theory that oculomotor mechanisms are, in turn, involved in programming movements of attention has received mixed support, but it appears to remain a viable hypothesis regarding the nature of the relationship between eye movements and attention.

\section{REFERENCES}

AllpORT, A. (1987). Selection-for-action: Some behavioral and neurophysiological considerations of attention and action. In $\mathbf{H}$. Heuer \& A. F. Sanders (Eds.), Perspectives on perception and action (pp. 395419). Hillsdale, NJ: Erlbaum.

AllPort, A. (1991). Visual attention. In M. I. Posner (Ed.), Foundations of cognitive science (pp. 631-682). Cambridge, MA: MIT Press.

BASHINSKI, H. S., \& BACHARACH, V. R. (1980). Enhancement of perceptual sensitivity as the result of selectively attending to spatial locations. Perception \& Psychophysics, 28, 241-280.

Cheal, M. L., \& Lyon, D. (1989). Attention effects on form discrimination at different eccentricities. Quarterly Journal of Experimental Psychology, 41A, 719-746.

Desimone, R., Wessinger, M., Thomas, L., \& Schneider, W. (1989). Effects of deactivation of lateral pulvinar or superior colliculus on the ability to selectively attend to a visual stimulus. Society for Neuroscience Abstracts, 15, 162.

DownING, C. J. (1988). Expectancy and visual-spatial attention: Effects on perceptual quality. Journal of Experimental Psychology: Human Perception \& Performance, 14, 188-202.

Eriksen, C. W., \& Hoffman, J. E. (1973). The extent of processing of 
noise elements during selective visual encoding from visual displays. Perception \& Psychophysics, 14, 155-160.

Eriksen, C. W., \& Hoffman, J. E. (1974). Selective attention: Noise suppression or signal enhancement? Bulletin of the Psychonomic Society, 4, 587-589.

GatTas, R., \& Desimone, R. (1992). Stimulation of the superior colliculus (SC) shifts the focus of attention in the macaque. Society for Neuroscience Abstracts, 18, 703

Guitton, D., Buchtel, H. A., \& Douglas, R. M. (1985). Frontal lobe lesions in man cause difficulties in suppressing reflexive glances and in generating goal-directed saccades. Exploratory Brain Research, 58, 455-472.

Hawkins, H. L., Hillyard, S. A., Luck, S. J., Mouloua, M., DownING, C. J., \& WoodWARD, D. P. (1990). Visual attention modulates signal detectability. Journal of Experimental Psychology: Human Perception \& Performance, 16, 802-811.

Henderson, J. M., Pollatsek, A., \& Rayner, K. (1989). Covert visual attention and extrafoveal information use during object identification. Perception \& Psychophysics, 45, 196-208.

HoFFMAN, J. E. (1975). Hierarchical stages in the processing of visual information. Perception \& Psychophysics, 18, 348-354.

Hoffman, J. E., \& Nelson, B. (1981). Spatial selectivity in visual search. Perception \& Psychophysics, 30, 283-290.

JUST, M. A., \& CARPENTER, P. A. (1987). The psychology of reading and language comprehension. Boston: Allyn \& Bacon.

KLEIN, R. (1980). Does oculomotor readiness mediate cognitive control of visual attention? In R. S. Nickerson (Ed.), Attention and performance VIII (pp. 259-276). Hillsdale, NJ: Erlbaum.

Klein, R. M., Kingstone, A., \& Pontefract, A. (1992). Orienting of visual attention. In K. Rayner (Ed.), Eye movements and visual cognition (pp. 46-65). New York: Springer-Verlag.

Klein, R. M., \& Pontefract, A. (1994). Does oculomotor readiness mediate cognitive control of visual attention? Revisited! In C. Umiltà \& M. Moskovitch (Eds.), Attention and performance XV (pp. 333350). Cambridge, MA: MIT Press.

KOWLER, E. (1985). Smooth eye movements as indicators of selective attention. In M. I. Posner \& O. S. M. Marin (Eds.), Mechanisms of attention: Attention and performance XI (pp. 285-300). Hillsdale, NJ: Erlbaum.

KOWLER, E. (1991). The role of visual and cognitive processes in the control of eye movement. In E. Kowler (Ed.), Eye movements and their role in visual and cognitive processes (pp. 1-70). Amsterdam: Elsevier.

Kowler, E., Anderson, E., Dosher, B., \& Blaser, E. (in press). The role of attention in the programming of saccades. Vision Research.

LYON, D. (1990). Large and rapid improvement in form discrimination accuracy following a location precue. Acta Psychologica, 73, 69-82.

McCoNkIE, G. W., \& RAYNER, K. (1975). The span of the effective stimulus during a fixation in reading. Perception \& Psychophysics, 17, 578-586.

MCCONKIE, G. W., \& RAYNER, K. (1976). Asymmetry of the perceptual span in reading. Bulletin of the Psychonomic Society, 8, 365-368.

PASHLER, H. (1989). Dissociations and dependencies between speed and accuracy: Evidence for a two-component theory of divided attention in simple tasks. Cognitive Psychology, 21, 469-514.

Pollatsek, A., Bolozky, S., Well, A. D., \& Rayner, K. (1981). Asymmetries in the perceptual span for Israeli readers. Brain \& Language, 14, 174-180.

POSNER, M. I. (1980). Orienting of attention. Quarterly Journal of Experimental Psychology, 32, 3-25.

Posner, M. I., Nissen, N. J., \& Ogden, W. C. (1978). Attended and unattended processing modes: The role of set for spatial location. In H. L. Picks \& I. J. Saltzman (Eds.), Modes of perceiving and processing information (pp. 137-157). Hillsdale, NJ: Erlbaum.
Rafal, R. F., Posner, M. I., Friedman, J. H., Inhoff, A. W., \& BernSTEIN, E. (1988). Orienting of visual attention in progressive supranuclear palsy. Brain, 111, 267-280.

RAYNER, K. (1975). The perceptual span and peripheral cues in reading. Cognitive Psychology, 7, 65-81.

Rayner, K., \& Pollatsek, A. (1989). The psychology of reading. Englewood Cliffs, NJ: Prentice Hall.

REMington, R. W. (1980). Attention and saccadic eye movements. Journal of Experimental Psychology: Human Perception \& Performance, 6, 726-744.

Remington, R. W., Johnston, J. C., \& Yantis, S. (1992). Involuntary attentional capture by abrupt onsets. Perception \& Psychophysics, $\mathbf{5 1}$ 279-290.

ReUTER-Lorenz, P. A., \& Fendrich, R. (1992). Oculomotor readiness and covert orienting: Differences between central and peripheral precues. Perception \& Psychophysics, 52, 336-344

Rizzolatti, G., Riggio, L., Dascola, I., \& Umiltà, C. (1987). Reorienting attention across the vertical and horizontal meridians: Evidence in favor of a premotor theory of attention. Neuropsychologia, 25, 31-40.

Rizzolatti, G., Riggio, L., \& Sheliga, B. M. (1994). Space and selective attention. In C. Umiltà \& M. Moskovitch (Eds.), Attention and performance $X V$ (pp. 231-265). Cambridge, MA: MIT Press.

SCHNeIDER, W. X., \& DeUbel, H. (1995). Visual attention and saccadic eye movements: Evidence for obligatory and selective spatial coupling. In J. M. Findlay, R. Kentridge, \& R. Walker (Eds.), Eye movement research: Mechanisms, processes, and applications (pp. 317-324). New York: Elsevier.

Shepherd, M., Findlay, J. M., \& Hockey, R. J. (1986). The relationship between eye movements and spatial attention. Quarterly Journal of Experimental Psychology, 38A, 475-491.

TAYlor, M. M., \& CReElman, C. D. (1967). PEST: Efficient estimates on probability functions. Journal of the Acoustical Society of America, 41, 782-787.

WurTz, R. H., Goldberg, M. E., \& Robinson, D. L. (1980). Behavioral modulation of visual responses in the monkey: Stimulus selection for attention and movement. Progress in Psychobiology \& Physiological Psychology, 9, 43-83.

Wurtz, R. H., GoldberG, M. E., \& Robinson, D. L. (1982). Brain mechanisms of visual attention. Scientific American, 246, 124-135. YARBUs, A. L. (1967). Eye movements and vision. New York: Plenum.

\section{NOTE}

1. It is important to establish that the effect of the attention cue on saccadic latency reported in Experiment 2 reflects the subjects' attempts to use the cue to direct attention rather than some automatic effect of the arrow. To test this, we ran an additional group of 7 subjects in an eyemovement control condition. In each of four blocks of 40 trials, the subjects made an eye movement to one of the four display positions. As in Experiment 2, an arrow pointing to one of the four positions was presented prior to the tone signaling the initiation of the saccade (see Figure 3). The direction of the arrow was random, and the subjects were instructed to ignore it. Average latencies were $234 \mathrm{msec}$ when saccade and arrow direction matched and $231 \mathrm{msec}$ when they were mismatched. A $t$ test showed this difference to be nonsignificant $[t(6)<1]$. This finding indicates that the increase in saccade latency when subjects are attempting to move their eyes and attention in different directions is due to voluntary attentional processes.

(Manuscript received June 21, 1993; revision accepted for publication January 20, 1995.) 